\title{
Molecular Communication on Artificial Cell Membranes (Invited Paper)
}

\author{
Jun-ichi Kikuchi \\ Masaru Mukai \\ Graduate School of Materials Science, Graduate School of Materials Science, \\ Nara Institute of Science and \\ Technology \\ Takayama, Ikoma, \\ Nara 630-0192, Japan \\ $+81-743-72-6090$ \\ jkikuchi@ms.naist.jp \\ Satoshi Hiyama \\ Research Laboratories, \\ NTT DOCOMO, Inc. \\ Hikarino-oka, Yokosuka, \\ Kanagawa 239-8536, Japan \\ +81-46-840-3811 \\ hiyama@nttdocomo.co.jp \\ Nara Institute of Science and \\ Technology \\ Takayama, Ikoma, \\ Nara 630-0192, Japan \\ +81-743-72-6093 \\ m-masaru@ms.naist.jp \\ Yuki Moritani \\ Research Laboratories, \\ NTT DOCOMO, Inc. \\ Hikarino-oka, Yokosuka, \\ Kanagawa 239-8536, Japan \\ +81-46-840-3811 \\ moritani@nttdocomo.co.jp
}

\begin{abstract}
Molecular communication is a bio-inspired communication paradigm using molecules as information carriers. In this paper, we built an example molecular communication system in aqueous media, which includes propagation of molecular capsules capable of carrying molecular information between a molecular sender and a molecular receiver and followed amplification of the information at the receiver. A gemini peptide lipid as a molecular switch embedded in the lipid bilayer membranes logically controlled propagation of molecular capsules formed with small liposomes from a sender to a receiver each composed of a giant liposome, with input signals, such as chemical, photonic, and thermal signals. In addition, the molecular switch acted as an artificial receptor at the receiver, receiving a photonic signal to communicate with an enzyme as a signal amplifier by using $\mathrm{Cu}^{2+}$ ion as a mediator between the receptor and the amplifier.
\end{abstract}

\section{Keywords}

Molecular communication, Artificial cell membrane, Molecular switch, Gemini peptide lipid, Enzymatic reaction.

\section{INTRODUCTION}

In biological system, cell membranes play pivotal roles as platforms for systematic transformation of materials, energy, and information. In order to simulate such biological functions, lipid

Permission to make digital or hard copies of all or part of this work for personal or classroom use is granted without fee provided that copies are not made or distributed for profit or commercial advantage and that copies bear this notice and the full citation on the first page. To copy otherwise, or republish, to post on servers or to redistribute to lists, requires prior specific permission and/or a fee.

BIONETICS'08, November 25-28, 2008, Hyogo, Japan.

Copyright 2008 ICST 978-963-9799-35-6. bilayer vesicles formed with phospholipids or synthetic lipids have been widely employed as cell membrane models [1-6]. While some biologically important events such as material transport, catalysis and energy conversion have been successfully simulated in the liposomal membranes [7-11], there have been few reports using lipid membranes as platforms for information processing.

Recently, we coined a word "molecular communication" which is a new communication paradigm using molecules as information carriers [12,13]. Information and communication technology highly developed in the last century now supports our daily life, however, there is growing interest to seek a novel communication technology which complements the present communication technology using electrons and photons as information carriers. The molecular communication inspired by the biological information processing would be one of the candidates. In general, the molecular communication system includes propagation of information-carrier molecules between a molecular sender and a molecular receiver and followed amplification of the information at the receiver.

In this paper, we built an example molecular communication system in aqueous media using the liposomal membranes. Figure 1 depicts a molecular communication system that we consider in the initial phase of our research. The present system is composed of a combination of the following unit processes; (1) sending of a molecular capsule which carries molecular information from a molecular sender, (2) receiving of the molecular capsule at a molecular receiver, and (3) amplification of molecular information at the receiver. The molecular capsules were formed with small liposomal membranes in a diameter range of 100-200 $\mathrm{nm}$. On the other hand, giant liposomes in the cell size (larger

\footnotetext{
${ }^{1}$ Research Laboratories, NTT DOCOMO, Inc.
} 
than $1 \mu \mathrm{m}$ ) were employed as the molecular sender and receiver. In order to realize selective propagation of the molecular capsules from the sender to the receiver, a gemini peptide lipid (GPL) was used as a photo-responsive molecular switch. When the molecular switch was embedded in liposomal membranes in the presence of transition metal ions, the propagation of the molecular capsules from the sender to the receiver was controlled by input signals such as chemical, photonic and thermal signals. The molecular switch at the receiver also acted as an artificial receptor, which tunes the activity of an enzyme as a chemical signal amplifier, by using a metal ion as a mediator between the receptor and the amplifier.

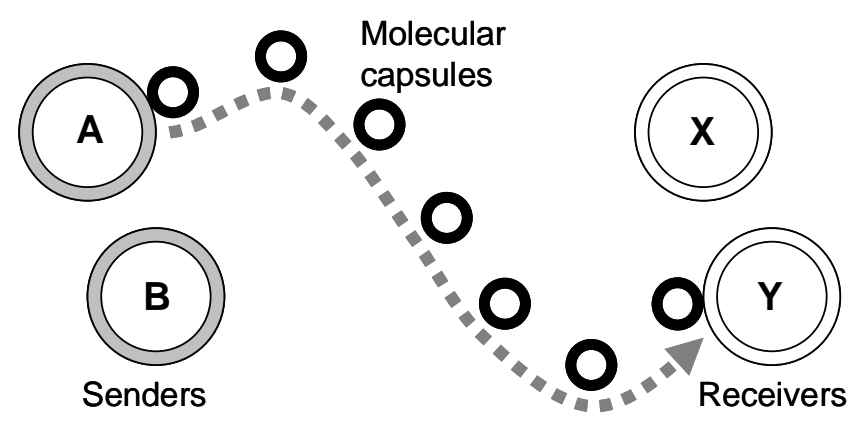

Figure 1. Molecular communication system composed of artificial cell membranes as senders, receivers and molecular capsules.

\section{DESIGN OF GEMINI PEPTIDE LIPID AS A MOLECULAR SWITCH}

Design of the molecular switch was inspired by naturallyoccurring gemini lipid, cardiolipin, having a unique dimeric lipid structure and interesting biological functions [14, 15]. Although much attention has been focused on physicochemical properties of synthetic gemini surfactants connecting two surfactant molecules via a spacer group [16-18], there are few reports on the synthetic gemini lipids with two double-chain segments like the cardiolipin. The molecular switch GPL is composed of three parts; two hydrophobic double-chain segments as hydrophobic anchors to the lipid membrane, two chiral histidyl residues as metal binding sites, and an azobenzene spacer unit as a photoresponsive part (Figure 2).

The space-filling models shown in Figure 3 represent plausible two conformations of the molecular switch embedded in the liposomal membranes. The conformations were minimized by molecular mechanics calculations using Cerius ${ }^{2}$ software based on the DREIDING force field [19]. Under thermodynamically stable conditions without photo-irradiation, the azobenzene moiety takes trans-form (trans-GPL); two imidazolyl groups in the gemini lipid are present over $0.6 \mathrm{~nm}$ apart from each other. Therefore, this species cannot act as a bidentate ligand for a metal ion. Upon ultraviolet (UV) light irradiation, the azobenzene moiety converts to the corresponding cis-form (cis- GPL) to be capable of forming a metal chelate that is effective for inducing the assembly of liposomes. Since cis-GPL reverts to trans-GPL by visible (VIS) light irradiation, the metal binding ability that is related to the

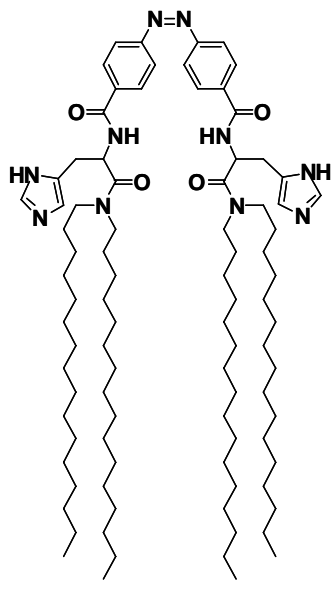

GPL

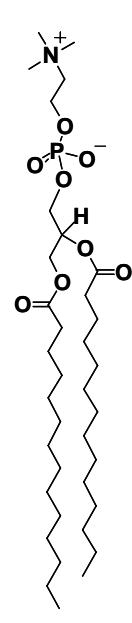

PL

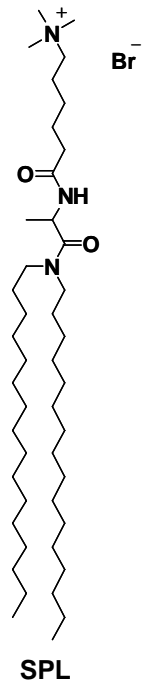

Figure 2. Molecular structures of gemini peptide lipid (GPL), phospholipid (PL) and synthetic peptide lipid (SPL).

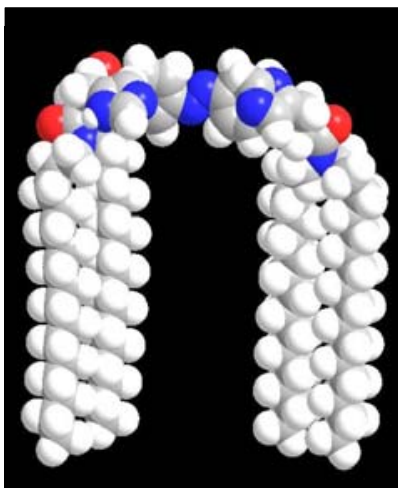

trans-GPL

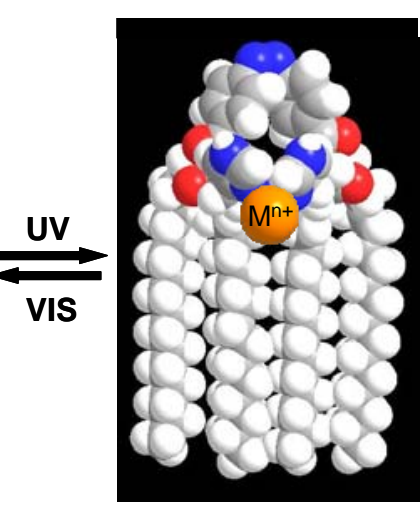

cis-GPL
Figure 3. Two conformations of the gemini peptide lipid generated upon photo-irradiation.

vesicular assembly would be changed through the photoisomerization reaction.

We have recently clarified that $\mathrm{Cu}^{2+}$-binding affinity of cis-GPL embedded in a liposomal membrane was much higher than that of the corresponding trans-form to give a photo-responsive assembling system of small liposomes [20]. We observed the liposomal assembly by means of freeze-fracture-replica transmission electron microscopy (TEM) and dynamic light scattering (DLS) measurements, however, fluorescence microscopy was unable to apply to visualize such assembly due to fluorescence quenching by $\mathrm{Cu}^{2+}$ ions. In the present work, $\mathrm{Cu}^{2+}$ ions were replaced by $\mathrm{Zn}^{2+}$ ions to visualize the photo-responsive dynamic propagation behavior of molecular capsules from a molecular sender to a molecular receiver. 


\section{PROPAGATION OF MOLECULAR CUPSULES}

Interaction of $\mathrm{Zn}^{2+}$ ions with molecular switch GPL embedded in the liposomal membrane formed with a phospholipid PL was evaluated by circular dichroism (CD) measurements. In general, metal-ligand interactions are much enhanced at the lipid membrane-water interface rather than in homogeneous aqueous solution. The CD spectra of the molecular switch embedded in the liposome were changed significantly upon addition of $\mathrm{Zn}^{2+}$ ions, reflecting the binding of $\mathrm{Zn}^{2+}$ ions to the L-histidyl residues of GPL. The titration isotherms were obtained by monitoring the CD intensity at $339 \mathrm{~nm}$ and $431 \mathrm{~nm}$ for cis-GPL and trans-GPL, respectively, which were applied to determine the binding constant of $\mathrm{Zn}^{2+}$ ions to the molecular switch. The Job's plot analyses for the liposomal system revealed that $\mathrm{Zn}^{2+}$ ions bind to the molecular switch in a ratio of 1:2. Thus, the binding constant for the 1:2 complex of $\mathrm{Zn}^{2+}$ ions with cis-GPL in the liposomes was evaluated to be $1.0 \times 10^{10} \mathrm{dm}^{6} \mathrm{~mol}^{-2}$. The value is comparable to the binding constant of $\mathrm{Cu}^{2+}$ ions with cis-GPL, $0.5 \times 10^{10} \mathrm{dm}^{6}$ $\mathrm{mol}^{-2}$ [20]. Contrastively, the binding constant for the zinc complex of trans-GPL in the liposome was $9.0 \times 10^{7} \mathrm{dm}^{6} \mathrm{~mol}^{-2}$, being much weaker than that for the corresponding cis-form. Accordingly, the metal-ligand interactions are effectively switched by photo-isomerization of the molecular switch embedded in the liposomal membranes.

In order to clarify functions of the photo-responsive molecular switch for propagation of molecular capsules, the photoresponsive assembling behavior of small liposomes equipped with GPL was evaluated by DLS measurements. Hydrodynamic diameter $\left(D_{\text {hy }}\right)$ of the liposomes containing cis-GPL with high metal-binding affinity was increased upon addition of $\mathrm{Zn}^{2+}$ ions, reflecting the liposomal assembly. On the other hand, the liposomes containing trans-GPL with much lower metal-binding affinity kept the $D_{\text {hy }}$ value nearly constant in the presence and absence of $\mathrm{Zn}^{2+}$ ions under the similar conditions. The results clearly indicate that difference in the metal binding ability between cis-GPL and trans-GPL in the lipid membrane markedly reflected on the photo-responsive assembly of liposomes.

On these grounds, control of the propagation of molecular capsules, formed with small liposomes, from a molecular sender to a molecular receiver, each composed of a giant liposome, was evaluated by means of phase-contrast and fluorescence microscopic observations. Initially, the molecular sender equipped with GPL in the cis-form anchors the molecular capsules bearing the same molecular switch in the presence of $\mathrm{Zn}^{2+}$ ions as schematically shown in Figure 4. Upon addition of a chemical signal having the metal binding ability much stronger than that of the molecular switch, such as ethylenediaminetetraacetate (EDTA), the molecular capsules were released from the molecular sender. The molecular capsules are also capable of sending, if the conformation of the molecular switches embedded in both the sender and the capsules changed from the cis-form to the trans-form through photo-isomerization. It is noteworthy that the sending behavior of the molecular capsules was also thermosensitive. That is, the molecular capsules were released from the molecular sender in the liquid crystalline state of the lipid membranes above the phase transition temperature $\left(T_{\mathrm{m}}\right)$ but not in the gel state below $T_{\mathrm{m}}$. The receiving process of the molecular capsules is in mirror image of the sending process. Thus, the molecular capsules having the molecular switch in the cis-form were selectively delivered onto the molecular receiver equipped with the same molecular switch in a temperature range above $T_{\mathrm{m}}$. On the other hand, trans-GPL derived by photo-isomerization of the corresponding cis-form did not perform as an effective molecular switch for receiving of the molecular capsules. The results indicate that propagation of the molecular capsules from the molecular sender to the molecular receiver was logically controlled by input signals, such as chemical, photonic, and thermal signals in the presence of the molecular switch.

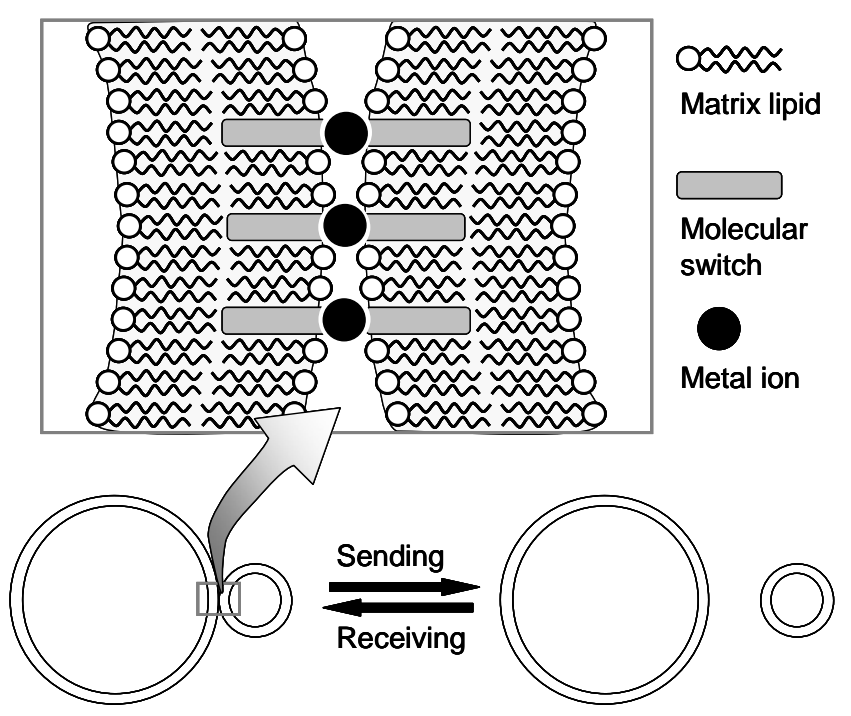

Figure 4. Intermembrane adhesion through metal complex formation of molecular switches embedded in liposomal membranes, which governs sending and receiving of a molecular capsule from a molecular sender and to a molecular receiver, respectively.

\section{AMPLIFICATION OF MOLECULAR INFORMATION}

As for amplification of molecular information at a molecular receiver, we employed artificial signal transduction system composed of a set of an artificial receptor, an enzyme, and a mediator between them. We have previously shown that catalytic activity of NADH-dependent lactate dehydrogenase (LDH) bound on a liposomal membrane was switched by an external signal input to an artificial receptor embedded in the membrane in the presence of $\mathrm{Cu}^{2+}$ ion as a mediator [21-26]. We chose here molecular switch GPL as an artificial receptor at the receiver. Thus, in the present signaling system, the enzymatic activity as a chemical signal output would be switched by photonic signal input into the receptor, while the previous receptors recognize chemical species as an input signal.

Amplification behavior of molecular information at a molecular receiver is schematically shown in Figure 5. Basic framework of the receiver was constructed with bilayer vesicle of a synthetic peptide lipid SPL. Photo-responsive molecular switch GPL as an artificial receptor and $\mathrm{LDH}$ as a signal amplifier were immobilized on the receiver membrane mainly through 
hydrophobic and electrostatic interactions, respectively. We have previously shown that the cationic bilayer vesicle provides an effective platform for immobilization of LDH with keeping its catalytic activity [27] and that $\mathrm{Cu}^{2+}$ ion performed as a potent competitive inhibitor for the enzyme bound on the cationic vesicle [28]. On the other hand, the molecular switch embedded in lipid bilayer membranes drastically changes $\mathrm{Cu}^{2+}$-binding affinity through photo-isomerization of the azobenzene moiety. Thus, at the molecular receiver equipped with the molecular switch and the enzyme, input of a photonic signal to the molecular switch would be converted to amplified chemical signal output at the enzyme through translocation of $\mathrm{Cu}^{2+}$ ion as a mediator between them.

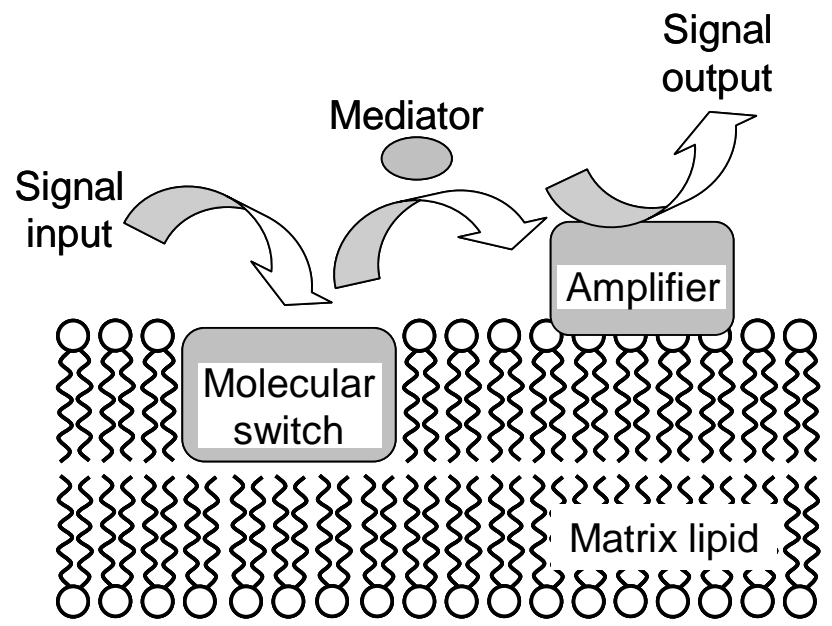

Figure 5. A flow of molecular information in amplification process at a molecular receiver.

LDH activities on the molecular receiver formed with synthetic peptide lipid SPL and molecular switch GPL were evaluated as a function of $\mathrm{Cu}^{2+}$ concentration. The enzymatic activity on the molecular receiver containing trans-GPL was drastically decreased with an increase of $\mathrm{Cu}^{2+}$ concentration, mainly reflecting binding of the metal ions to the enzyme active site, since the metal-binding ability of the molecular switch in the trans-form is much lower than that of the enzyme. On the other hand, the enzymatic activity on the molecular receiver containing cis-GPL showed higher value, as compared with that on the molecular receiver containing trans-GPL. The results clearly indicate that signal amplification as an enzymatic reaction on the molecular receiver is switchable through photo-isomerization of the molecular switch.

The present molecular receiver was highly specific to metal ions as the mediator species between the molecular switch and the enzyme. LDH activities on the receiver formed with synthetic peptide lipid SPL containing molecular switch GPL in the cisform or the trans-form was evaluated in the presence and absence of various metal ions. While the enzymatic activity on the receiver was markedly switched through photo-isomerization of trans-GPL to cis-GPL in the presence of $\mathrm{Cu}^{2+}$ ions, other metal ions such as $\mathrm{Zn}^{2+}, \mathrm{Ni}^{2+}$ and $\mathrm{Co}^{2+}$ did not perform as effective mediators between the molecular switch and the enzyme. The molecular switch has analogous binding affinity toward $\mathrm{Cu}^{2+}$ and
$\mathrm{Zn}^{2+}$ ions, whereas the enzymatic activity was specifically inhibited by $\mathrm{Cu}^{2+}$ but not by $\mathrm{Zn}^{2+}$ ions. Thus, $\mathrm{Cu}^{2+}$ ion exhibiting strong affinity toward both the molecular switch and the enzyme acts as a specific mediator in the present molecular receiver system.

In order to clarify the structural importance of the gemini peptide lipid having an azobenzene moiety and two L-histidyl residues as the photo-responsive molecular switch, molecular switch GPL was replaced by three reference compounds; non-gemini peptide lipid with an L-histidyl residue, gemini peptide lipid with an azobenzene spacer unit but without two imidazolyl groups, and gemini peptide lipid with two L-histidyl residues but without an azobenzene moiety [29]. LDH activities on the molecular receiver formed with synthetic peptide lipid SPL containing these molecular switch analogs were evaluated upon UV or visible light irradiation. The results clearly indicate that the gemini structure with both an azobenzene spacer unit and two L-histidyl residues is essential to perform as the photo-responsive molecular switch to control the enzymatic activity on the molecular receiver.

\section{CONCLUSION}

It became apparent that a bio-inspired molecular communication system was constructed by using lipid bilayer membranes. The system includes two processes, that is, propagation of molecular capsules carrying molecular information from a molecular sender to a molecular receiver and amplification of the molecular information at the receiver. Both processes were controlled by a common molecular switch embedded in the lipid membranes. Thus, propagation of molecular capsules was controlled by chemical, photonic, and thermal signals. In addition, signal amplification as an enzymatic reaction was effectively switched on a molecular receiver through intermolecular communication between a molecular switch and an enzyme, using photonic stimulus and a metal ion as an input signal and a mediator, respectively. Although the present work is regarded as the initial phase of research in molecular communication, various kinds of molecular communication systems would be imaginable on the basis of our research results. We believe that further extensive study aiming to develop the molecular communication system will promise to open the door for an interdisciplinary area between bio-nanotechnology and information and communication technology.

\section{REFERENCES}

[1] Barenholz, Y. Liposome Applications: Problems and Prospects, Curr. Opin. Colloid Interface Sci. 2001, 6, 66-77.

[2] Lian, T.; Ho, R. J. Y. Trends and Developments in Liposome Drug Delivery Systems, J. Pharm. Sci. 2001, 90, 667-680.

[3] Monnard, P.-A. Liposome-entrapped Polymerases as Molels of Microscale/Nanoscale Bioreactors, J. Membrane Biol. 2003, 191, 87-97.

[4] Kunitake, T. Synthetic Bilayer Membranes: Molecular Design and Molecular Organization, In Comprehensive Supramolecular Chemistry, Vol. 9; Atwood, J. L., Davies, J. E. 
D., MacNicol, D. D., Vögtle, F., Lehn, J. M., Eds.; Pergamon Press: Oxford, 1996; pp 351-406.

[5] Murakami, Y.; Kikuchi, J. Supramolecular Assemblies Formed with Synthetic Peptide Lipids. Functional Models of Biomembranes and Enzymes, In Bioorganic Chemistry Frontiers, Vol. 2; Dugas, H., Ed.; Springer-Verlag: Berlin, 1991; pp 73-113.

[6] Mueller, A.; O’Brien, D. F. Supramolecular Materials via Polymerization of Mesophases of Hydrated Amphiphiles, Chem. Rev. 2002, 102, 727-757.

[7] Mitchell, K. D. D.; Fyles, T. M. Ion Channels and Their Models, In Encyclopedia of Supramolecular Chemistry, Vol. 1; Atwood, J. L., Steed J. W., Eds.; Marcel Dekker: New York, 2004; pp 742-746.

[8] Smith, B. D.; Lambert, T. N. Molecular Ferries: Membrane Carriers That promote Phospholipid Flip-Flop and Chloride Transport, Chem. Commun. 2003, 2261-2268.

[9] Murakami, Y.; Kikuchi, J.; Hisaeda, Y.; Hayashida, O. Artificial Enzymes, Chem. Rev. 1996, 96, 721-758.

[10] Gust, D.; Moore, T. A.; Moore, A. L. Mimicking Photosynthetic Solar Energy Transduction, Acc. Chem. Res. 2001, 34, 40-48.

[11] Kikuchi, J.; Sasaki, Y.; Ariga, K. Molecular Recognition and Functional Connection in Lipid Membranes, In Advances in Supramolecular Chemistry, Vol. 8; Gokel G. W., Ed.; Cerberus Press: South Miami, 2002; pp 131-173.

[12] Hiyama, S.; Moritani, Y.; Suda, T.; Egashira, R.; Enomoto, A.; Moore, M.; Nakano, T. Molecular Communication, Proc. NSTI Nanotechnology Conference and Trade Show 2005 (Nanotech'05), 2005, 3, 391-394.

[13] Moritani, Y.; Hiyama, S.; Suda, T. Molecular Communication - A Biochemically-Engineered Communication System-, Proc. Frontiers in the Convergence of Bioscience and Information Technologies 2007 (FBIT'07), 2007, 839-844.

[14] Hoch, F. L. Cardiolipins and Biomembrane Function, Biochim. Biophys. Acta 1992, 1113, 71-133.

[15] Schlame, M.; Rua, D.; Greenberg, M. L. The Biosynthesis and Functional Role of Caldiolipin, Prog. Lipid Res. 2000, 39, 257-288.

[16] Menger, F. M.; Keiper, J. S. Gemini Surfactants, Angew. Chem. Int. Ed. 2000, 39, 1906-1920.

[17] Zana, R. Dimeric (Gemini) Surfactants: Effect of the Spacer Group on the Association Behavior in Aqueous Solution, $J$. Colloid Interface Sci. 2002, 248, 203-220.
[18] Zana, R.; Xia, J., Eds. Gemini Surfactants; Marcel Dekker: New York, 2004.

[19] http://accelrys.com/products/cerius2/

[20] Sasaki, Y.; Iwamoto, S.; Mukai, M.; Kikuchi, J. Photo- and Thermo-responsive Assembly of Liposomal Membranes Triggered by a Gemini Peptide Lipid as a Molecular Switch, $J$. Photochem. Photobiol., A 2006, 183, 309-314.

[21] Kikuchi, J.; Ariga, K.; Ikeda, K. Signal Transduction Mediated by Artificial Cell-surface Receptors: Activation of Lactate Dehydrogenase Triggered by Molecular Recognition and Phase Reorganization of Bile Acid Derivatives Embedded in a Synthetic Bilayer Membrane, Chem. Commun. 1999, 547548.

[22] Kikuchi, J.; Ariga, K.; Miyazaki, T.; Ikeda, K. An Artificial Signal Transduction System. Control of Lactate Dehydrogenase Activity Performed by an Artificial Cellsurface Receptor, Chem. Lett. 1999, 253-254.

[23] Fukuda, K.; Sasaki, Y.; Ariga, K.; Kikuchi, J. Dynamic Behavior of a Transmenbrane Molecular Switch as an Artificial Cell-surface Receptor, J. Mol. Catal. B 2001, 11, 971-976.

[24] Tian, W.-J.; Sasaki, Y.; Ikeda, A.; Kikuchi, J.; Fan, S.-D. Intermolecular Communication on Lipid Bilayer Membranes. Control of Enzymatic Activity Triggered by a Lipid Signal, Chem. Lett. 2004, 33, 226-227.

[25] Tian, W.-J.; Sasaki, Y.; Fan, S.-D.; Kikuchi, J. Switching of Enzymatic Activity through Functional Connection of Molecular Recognition on Lipid Bilayer Membranes, Supramol. Chem. 2005, 17, 113-119.

[26] Tian, W.-J.; Sasaki, Y.; Fan, S.-D.; Kikuchi, J. Intermolecular Communication on Lipid Bilayer Membrane. Tuning of Enzymatic Activity with Phase Transition of the Matrix Membranes, Bull. Chem. Soc. Jpn. 2005, 78, 715-717.

[27] Kikuchi, J.; Kamijyo, Y.; Etoh, H.; Murakami, Y. Catalytic Performance of a Supramolecular Bienzyme Complex Formed with Artificial Aminotransferase and Natural Lactate Dehydrogenase, Chem. Lett. 1996, 427-428.

[28] Kikuchi, J.; Ariga, K.; Miyazaki, T.; Ikeda, K. An Artificial Signal Transduction System. Control of Lactate Dehydrogenase Activity Performed by an Artificial Cellsurface Receptor, Chem. Lett. 1999, 253-254.

[29] Iwamoto, S.; Otsuki, M.; Sasaki, Y.; Ikeda, A.; Kikuchi, J. Gemini Peptide Lipids with Ditopic Ion-recognition Site. Preparation and Functions as an Inducer for Assembling of Liposomal Membranes, Tetrahedron 2004, 60, 9841-9847. 